\title{
The interactive web-based program MSmonitor for self-management and multidisciplinary care in multiple sclerosis: concept, content, and pilot results
}

\author{
This article was published in the following Dove Press journal: \\ Patient Preference and Adherence \\ II December 2015 \\ Number of times this article has been viewed
}

Peter Joseph Jongen, ${ }^{1,2}$ Ludovicus G Sinnige, ${ }^{3}$ Björn M van Geel, ${ }^{4}$ Freek Verheul, ${ }^{5}$ Wim I Verhagen, ${ }^{6}$ Ruud A van der Kruijk, ${ }^{7}$ Reinoud Haverkamp, ${ }^{8}$ Hans M Schrijver, ${ }^{9}$ J Coby Baart, ${ }^{10}$ Leo H Visser, ${ }^{\prime \prime}$ Edo P Arnoldus, ${ }^{12} \mathrm{H}$ Jacobus Gilhuis, ${ }^{13}$ Paul Pop, ${ }^{14}$ Monique Booy, ${ }^{15}$ Wim Lemmens, ${ }^{16}$ Rogier Donders, ${ }^{16}$ Anton Kool, ${ }^{17}$ Esther van Noort ${ }^{17}$

'Department of Community and Occupational Medicine, University Medical Center Groningen, University Groningen, Groningen, ${ }^{2}$ MS4 Research Institute, Nijmegen, ${ }^{3}$ Multiple Sclerosis Centre Leeuwarden, Medical Centre Leeuwarden, Leeuwarden, ${ }^{4}$ Department of Neurology, Medical Centre Alkmaar, Alkmaar, ${ }^{5}$ Department of Neurology,

Groene Hart Hospital, Gouda, ${ }^{6}$ Department of Neurology, Canisius Wilhelmina Hospital, Nijmegen, ${ }^{7}$ Department of Neurology,

Slingeland Hospital, Doetinchem,

${ }^{8}$ Department of Neurology, Zuwe

Hofpoort Hospital, Woerden, ${ }^{9}$ Multiple Sclerosis Centre, Westfries Gasthuis,

Hoorn, ${ }^{10}$ Department of Neurology,

Ziekenhuisgroep Twente, Almelo-Hengelo,

"Multiple Sclerosis Centre Midden Brabant,

St Elisabeth Hospital, ${ }^{12}$ Multiple Sclerosis

Centre Midden Brabant, Tweesteden

Hospital, Tilburg, ${ }^{13}$ Department of Neurology, Reinier de Graaf Gasthuis,

Delft, ${ }^{14}$ Department of Neurology, Viecuri

Medical Centre, Venlo-Venray, ${ }^{15}$ Multiple

Sclerosis Centre, Amphia Hospital, Breda,

${ }^{16}$ Department for Health Evidence, Radboud

University Nijmegen Medical Centre,

Nijmegen, ${ }^{17}$ Curavista bv, Geertruidenberg, the Netherlands

Correspondence: Peter Joseph Jongen Department of Community and

Occupational Medicine, University Medical

Center Groningen, University Groningen,

Antonius Deusinglaan I, 9713 AV Groningen,

the Netherlands

Email p.j.h.jongen@rug.nl
Background: There is a growing need to offer persons with multiple sclerosis (PwMS) possibilities for self-management and to integrate multidisciplinary health data. In 2009-2014 we developed a patient-reported outcome based, interactive, web-based program (MSmonitor) for (self-)monitoring, self-management and integrated, multidisciplinary care in MS.

Methods: The notions underlying the MSmonitor concept and the program's elements are described. We analyze MSmonitor's role in the self-management of fatigue by retrospective comparison of fatigue and health-related quality of life (HRQoL) before and after usage of specific elements of MSmonitor, and by a correlative analysis between frequency of usage and fatigue change.

Results: After a step-wise development the program comprises six validated questionnaires: Multiple Sclerosis Impact Profile, Modified Fatigue Impact Scale-5 items (MFIS-5), Hospital Anxiety and Depression Scale, Multiple Sclerosis Quality of Life-54 items, and the 8-item Leeds Multiple Sclerosis Quality of Life (LMSQoL) questionnaires; two inventories: Medication and Adherence Inventory, Miction Inventory; two diaries: Activities Diary, Miction Diary; and two functionalities: e-consult and personal e-logbook. The program is now used in 17 hospitals by 581 PwMS and their neurologists, MS nurses, physical therapists, rehabilitative doctors, continence nurses, and family doctors. Those PwMS (N=105) who used the LMSQoL and MFIS-5 questionnaires at least twice in a period of up to 6 months, showed improved HRQoL $(P<0.026)$. In the subgroup ( $\mathrm{N}=56$ ) who had also used the Activities Diary twice or more, the frequency of diary usage correlated modestly with the degree of fatigue improvement ( $r=0.292 ; P=0.028$ ).

Conclusion: MSmonitor is an interactive web-based program for self-management and integrated care in PwMS. Pilot data suggest that the repeated use of the short MFIS-5 and LMSQoL questionnaires is associated with an increase in HRQoL, and that a repeated use of the Activities Diary might contribute to the self-management of fatigue.

Keywords: multiple sclerosis, self-management, web-based, care, multidisciplinary, patientreported outcome

\section{Introduction}

Multiple sclerosis (MS) is a chronic inflammatory and degenerative disease of the central nervous system. In approximately $80 \%$ of the persons with multiple sclerosis (PwMS) the first clinical phase is characterized by recurrent episodes of new or worsening symptoms (relapses), which are typically followed by complete or partial remissions: relapsing remitting MS (RRMS). Anti-inflammatory immune-modulating or immune-suppressing drugs may substantially reduce the frequency and severity of relapses, and thus modify the 
disease course of RRMS. However, the long-term outcome of disease modifying drug (DMD) treatment is largely unknown. After 10-15 years most persons with RRMS convert to the secondary progressive (SP) phase. It has not been elucidated to what degree DMDs may prevent or postpone the conversion to SPMS. The SP phase is characterized by a slow but relentless increase in disability and rare or absent relapses. Approximately 15\% of PwMS have a progressive course from disease onset: primary progressive MS. In both SPMS and primary progressive MS DMDs are not effective.

Epidemiological studies in RRMS indicate that the interval from disease onset to the occurrence of irreversible major disabilities (walking with aid, being wheelchair bound) depends on the time interval between disease onset and the attainment of lower disability levels (limited walking but without aid); and that the time to reach these lesser disabilities depends on the inflammatory disease activity in the first 2 years after diagnosis. ${ }^{1-3}$ This means that there is an early and limited therapeutic window, and that to optimally prevent long-term disability, DMDs should be used strategically and efficiently. This requires a close clinical monitoring, in order to assess any increase in disability, due to incomplete recovery of relapses, or any worsening of health-related quality of life (HRQoL), an overall subjective measure of effectiveness, as well as to detect side effects. ${ }^{4,5}$ The requirements for intense monitoring may not be fully met by doctor-centered measurements during regular outpatient visits. More frequent assessments and the use of patient-reported outcomes (PROs) could have an added value. , $^{6,7}$

In MS, the lesions in the central nervous system are multifocal and widespread. Depending on the actual localization of lesions PwMS may experience a great variety of symptoms and signs. Therefore, MS treatment is typically multidisciplinary, involving medical specialists, specialized nurses, and other paramedical disciplines. Thus, the multidisciplinary team of health care professionals may include a neurologist, registered nurse specializing in MS, psychologist, physical therapist, occupational therapist, ophthalmologist, rehabilitation specialist, dietician, gastroenterologist, and others. Moreover, as it is largely unpredictable whether or when a specific problem may occur, MS care and counseling should be flexible and personalized.

In PwMS the accumulation of disabilities leads to the loss of independence, which in turn may negatively affect autonomy and self-efficacy. ${ }^{8-10}$ Low self-efficacy has been associated with lower HRQoL ${ }^{11,12}$ and less physical activity. ${ }^{12}$ Since a lowered self-efficacy causes PwMS to undervalue their actual capabilities, a vicious circle may start and still existing functions run a risk to eventually disappear. Self-efficacy may be increased via self-management. In general, self-management comprises interventions, education, training, and skills by which patients with a chronic condition, disability, or disease can effectively take care of themselves and learn how to do so. ${ }^{13}$ Given that the onset of MS is often in early adulthood, PwMS could substantially benefit from early adopted self-management strategies.

In view of the aforementioned considerations we set out to develop an interactive web-based program for selfmanagement and professional multidisciplinary care in MS, and we explored the program's role in self-management.

\section{Methods}

\section{Concept}

The concept of the MSmonitor program is based on three notions: the autonomy of PwMS; the multidisciplinary character of MS care; and a collaboration of the stakeholders involved in MS care.

\section{Autonomy of PwMS}

Autonomy is the capacity to make informed un-coerced decisions, and is the basis of the responsibility and accountability for one's actions. In PwMS, autonomy is the basis for self-management and shared decision making. By facilitating self-assessments, self-monitoring, and self-management, the MSmonitor program aims to utilize and increase PwMS' autonomy. The fact that PwMS own their personal data generated by MSmonitor and decide on which health care professional to authorize access to the data, is in line with autonomy as a leading principle of MSmonitor.

\section{Multidisciplinary care}

From the PwMS' perspective, health care is not organized to efficiently provide multidisciplinary care. In daily practice the communication between health care professionals may be inadequate, leading to deficient quality of care or repetition of measurements. By making the self-assessments' results available to all authorized members of the multidisciplinary team, the MSmonitor program prevents unnecessary assessments and enables an optimal use of PROs. Moreover, the e-consult functionality facilitates communication between PwMS and health care professionals, and between professionals mutually.

\section{Collaboration of stakeholders}

MSmonitor has been developed step by step by the immaterial and material input of various stakeholders: 1) PwMS, 
2) health care professionals, 3) hospitals, 4) a software company (Curavista bv), 5) a research institute (MS4 Research Institute), 6) a Scientific Advisory Board, and 7) funding partners (TEVA, Sanofi-Aventis). The synergy of the collaboration between PwMS, professionals, and public and private organizations results from their combined respected expertise and input. The collaboration is dynamic and developing, has a minimum of formalization - which promotes flexibility and creativity - and is based on the endorsement by each participant of the MSmonitor concept. The MSmonitor Working Group includes all neurologists and MS nurses that use MSmonitor. Curavista bv ( $\mathrm{AK}, \mathrm{EvN})$ is a commercial software company specializing in health applications and online assessments in acute and chronic health disorders. The MS4 Research Institute (PJJ) is an independent scientific not-for-profit organization dedicated to e-health research, especially in MS. TEVA and Sanofi-Aventis are pharmaceutical companies manufacturing DMDs.

The aspects to be monitored and the program's elements were suggested by PwMS, neurologists, and nurses. Which tool to use for assessing a certain aspect, was primarily suggested by neurologists and nurses, based on their experiences and preferences. Then, the MS4 Research Institute checked the psychometric validity of the suggested tool, based on a review of the literature. The final choice was made by the MSmonitor Working Group and approved by the Scientific Advisory Board. The members of the Scientific Advisory Board are Stephan T Frequin $\mathrm{PhD}$, neurologist (St Antonius Hospital, Nieuwegein, the Netherlands), Dianne Groenewoud, epidemiologist (Nijmegen, the Netherlands), Kitty Harrison RN MANP, MS nurse (Tergooi Ziekenhuizen, Blaricum, the Netherlands) and Dorothea Heersema $\mathrm{PhD}$, neurologist (University Medical Centre Groningen, Groningen, the Netherlands).

\section{Content and availability}

At the time of study (2014) MSmonitor's content comprised nine components and two functionalities in five categories: 1) questionnaires, 2) inventories, 3 ) diaries, 4) e-consult, and 5) personal e-logbook. In line with the autonomy principle, it was not predefined which patients should use what part of the program and when. The actual use of the various elements differed between the centers and also depended on the neurologists' and nurses' preferences. For example, some centers focused on the use of the Multiple Sclerosis Impact Profile (MSIP) for their regular outpatient consultations, others on the use of the Miction Inventory.

\section{Questionnaires}

The questionnaires are psychometrically validated and include the MSIP, Multiple Sclerosis Quality of Life-54 (MSQoL-54), Modified Fatigue Impact Scale-5 Item Version (MFIS-5), Leeds Multiple Sclerosis Quality of Life (LMSQoL), and the Hospital Depression Anxiety Scale (HADS).

The MSIP comprises 36 questions assessing disability (Q1a-Q36a) and disability perception (Q1b-Q36b) in seven domains (muscle and movement functions, excretion and reproductive functions, basic movement activities, activities of daily living, participation in life situations, environmental factors, mental functions) and four symptoms (fatigue, pain, speech, vision). ${ }^{14-16}$ In addition to yielding validated domain and symptom scores, the MSIP provides a complete overview of MS-related symptoms and their subjective valuation. Completion time is approximately 20 minutes.

The MSQoL-54 provides an MS-specific, multidimensional assessment of patient-centered health status, and consists of the Short Form 36-Item health survey as a generic core measure, supplemented with 18 questions on items relevant to PwMS in the areas of health distress, sexual function, satisfaction with sexual function, overall QoL, cognitive function, energy, pain and social function. ${ }^{17}$ The MSQoL-54 contains 52 items distributed into 12 scales, and two single items. A physical and a mental dimension underlie the MSQoL-54: the physical and mental domains. ${ }^{17}$ Completion time is approximately 15 minutes.

The MFIS-5 is a short, 5-item questionnaire examining a patient's perceived impact of fatigue on a variety of daily activities over the past month. ${ }^{18}$ Answers to each question are rated on a 5-point scale from 0 to 4 . The MFIS-5 total score consists of the sum of the raw scores on these five items and ranges from 0 to 20, where higher scores indicate more experienced fatigue. ${ }^{18}$ Completion time is approximately 2 minutes.

The LMSQoL is a self-assessment scale that consists of eight questions, examining MS-related aspects of QoL over the past month. ${ }^{19,20}$ Answers are rated on a 4-point scale from 0 to 3 an the resulting score ranges from 0 to 24, with higher scores reflecting higher levels of well-being. Completion time is approximately 2 minutes.

The HADS is a 14-item, self-report questionnaire for anxiety and depression. ${ }^{21}$ The HADS consists of two subscales, one for anxiety and one for depression, each comprising seven questions. Completion time is approximately 5 minutes.

For all questionnaires scores are automatically generated by the program and presented in graphs and tables to PwMS and their authorized caregivers. 


\section{Inventories}

Inventories provide complete overviews and do not generate scores. The Miction Inventory consists of an overview of urological symptoms known to occur in MS. The Medication and Adherence Inventory gives a patient-reported update of medication that is taken, the number of missed DMD doses in the last month, and the date and reason of an eventual DMD discontinuation.

\section{Diaries}

Diaries are meant to give insight into MS-related processes over 24-hour periods. The Activities Diary records the type and duration of activities and rest periods. The Miction Diary documents the frequencies and quantities of mictions and fluid intakes.

\section{E-consult}

The e-consult functionality enables PwMS to contact their health care professionals, to ask questions or provide feedback, and enables professionals to communicate mutually.

\section{E-logbook}

The e-logbook functionality is a personal e-diary for recording items or experiences that are not covered by the standardized questions of the questionnaires or inventories. It has the option to set personal goals for self-management.

\section{Standard availability}

The MSIP, MSQoL-54, MFIS-5, and LMSQoL questionnaires, Medication and Adherence Inventory, diaries, e-consult, and e-logbook are available to all participating PwMS. The MFIS-5, LMSQoL, and Medication and Adherence Inventory can be used monthly; the MSIP at least 6-monthly and at most monthly (depending on the choice of the center); the MSQoL-54 annually; the diaries and e-logbook daily; and the e-consult whenever needed.

The use of LMSQoL, MFSI-5, and Medication and Adherence Inventory ("Quickscan”) enables quick monthly assessments of one's HRQoL, experienced fatigue, medication, and adherence.

\section{Selective availability}

The completions of the HADS and Miction Inventory are indicated by the health care professionals. The HADS is made available by neurologist, MS nurse or psychologist on clinical indication, eg, when suspecting an anxiety or mood disorder, or for evaluating the effectiveness of a treatment. In case of a sub-score $>8$, an alert is presented on the dashboard to warn about the abnormal finding. The Miction Inventory is made available by neurologist, MS nurse, urologist or continence nurse on indication.

\section{Recent developments}

A recent user of the MSmonitor program is the not-for-profit organization MS Care Netherlands (MS Zorg Nederland). One of the activities of MS Care Netherlands is the development of regional multidisciplinary networks of qualified health care professionals in the field of MS. Its primary goal is that optimal multidisciplinary professional care for PwMS is available locally and in every phase of the disease. To this end, MS Care Netherlands organizes regional training, education, and certification of health care professionals, and develops multidisciplinary guidelines for MS care processes. The first five regional networks started in October 2013 with 200 health care professionals being trained in providing optimal care for approximately 2,000 PwMS. In 2014, five additional regional networks started, bringing the total of certified health care professionals to 400 . The ultimate goal of MS Care Netherlands is to establish multidisciplinary regional networks in every region of the Netherlands. The use of the MSmonitor program is thought to facilitate the extramural implementation of multidisciplinary and personalized treatment programs via exchange of information and sharing PROs.

Recently the applications of the short questionnaires, e-consult, and diaries for smart phones have been implemented (Figure 1). The mobile use of selected components is thought to improve the self-management, screening, and communication options of the program. The full use, including longer questionnaires, is conceivably best realized via tablets, laptops or desktops. It is of note that in PwMS motor, sensory, coordinative, or visual symptoms may interfere with smart phone use and thus limit the applicability of the mobile option.

The prevalence of urological symptoms is high in MS, affecting $80 \%-100 \%$ of the PwMS during the course of the disease. ${ }^{22}$ In 2013 a self-screening tool for neurogenic bladder overactivity (Actionable questionnaire) was developed. ${ }^{23,24}$ This 8 -item questionnaire is a reliable, sensitive, and specific screening tool for people with bladder problems related to $\mathrm{MS}^{23,24}$ It may be especially useful in identifying PwMS who could benefit from further urologic evaluation and treatment. ${ }^{23,24}$ In 2015, a Dutch version of the Actionable questionnaire was validated. We imagine that PwMS screen themselves online on a regular basis or after the occurrence of (new) bladder symptoms. 


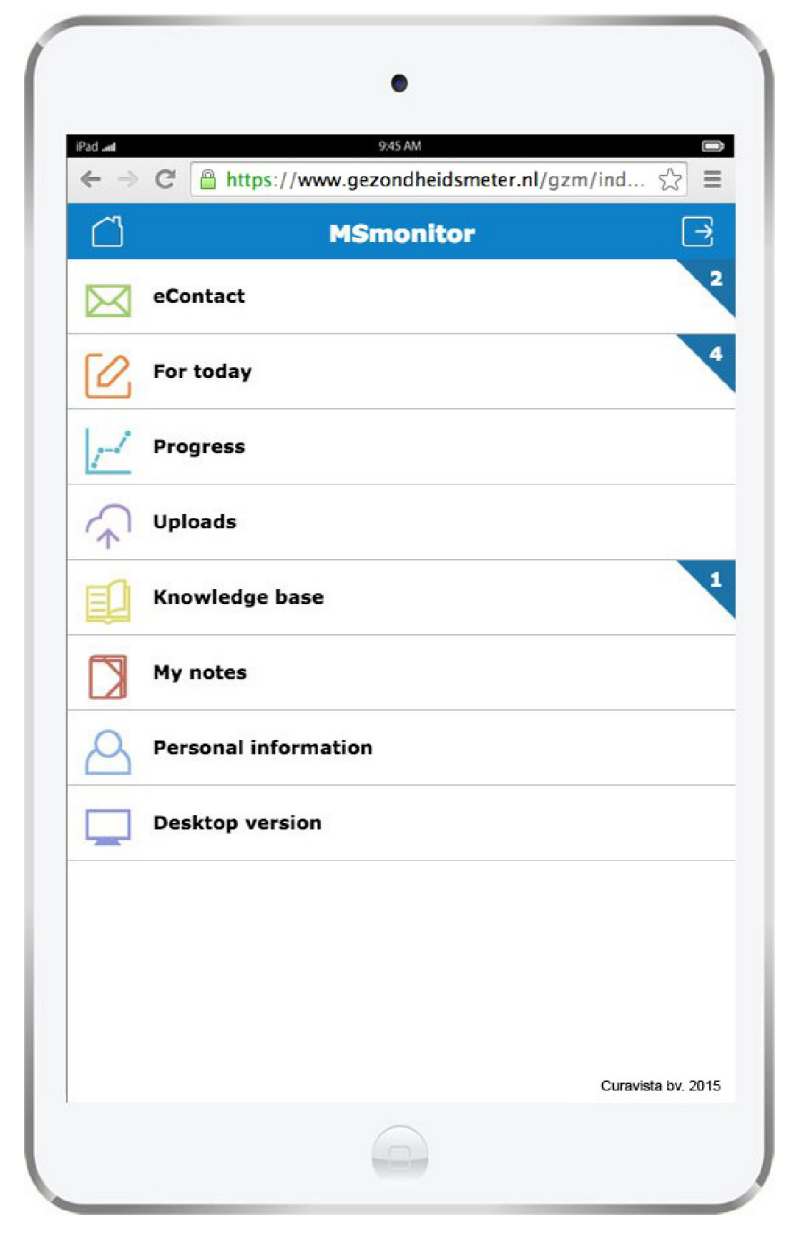

Figure I Screenshot of MSmonitor patient dashboard for iOS and Android smart phones.

A positive screening may be followed by an e-consult, completion of the Miction Diary and Miction Inventory or self-management.

The neurological assessment of disabilities is essential to the monitoring of MS disease activity. The Expanded Disability Status Scale (EDSS) is widely used in clinical studies and neurological practice. ${ }^{25,26}$ The EDSS is a single, ordinal, non-linear composite score based on observerrated scales in seven neurological functional systems, and ambulation. ${ }^{26}$ In 2013, the Medisch Centrum Leeuwarden started the implementation of the Treatment Optimization Recommendations of the Canadian Multiple Sclerosis Working Group. ${ }^{27}$ This gave rise to the inclusion of the EDSS in the MSmonitor program.

With the growing numbers of participating hospitals and PwMS the quantity of patient-reported data is rapidly increasing. Actually MSmonitor counts 1,500 visits per month and 150 completed forms per week. Consequently, we envisage to analyze the MSmonitor data base for research purposes. Moreover, PwMS may be contacted by their neurologist or nurse via the "mail-to-all" function, to be informed about a study or survey. Persons who want to participate confirm their participation online after having read the informed consent page, and fill in the research questionnaire. Additional patient-reported data, eg, on relapses, can be obtained in the context of study protocols.

\section{Technical, security, and ethical aspects}

The MSmonitor program is a modular application on the Curavista e-Health Platform, built on an Oracle database with JAVA-scripting, XML-applets and AJAX protocols. Data processing is 256-bits encrypted with VPN-tunneling. The databases are physically and software secured in a dedicated data center in the Netherlands. Prior to participation PwMS give electronic consent for storage of personal data and their responses in the database, for access to data by attending health care professionals of their choice, and for analyses of anonymized and aggregated data for scientific or policy purposes (eg, to improve the content of MSmonitor). The reasons for data analyses are checked by an independent supervisory board (Stichting Infometer). It is possible for PwMS to participate in MSmonitor without giving consent for data analyses. The MSmonitor project and the protocol for analysis of the pilot data were not reviewed by an ethical committee, as they did not qualify for being tested according to the Dutch Medical Research Involving Human Subjects Act of 1999 (http://wetten.overheid.nl/BWBR0009408). ${ }^{28}$

\section{Data analysis}

To explore the potential value of the MSmonitor program with respect to the self-management of fatigue, we analyzed the relationships between the levels of fatigue (MFIS-5) and HRQoL (LMSQoL) on the one hand, and the usage of the Activities Diary on the other. We hypothesized, first, that the usage of the diary was associated with a decrease in fatigue and an increase in HRQoL; second, that the frequency of diary usage was associated with the degree of fatigue decrease and HRQoL increase; and, third, that the mere use of the MFIS-5 and LMSQoL (self-assessment per se) in the Quickscan was associated with an increase in HRQoL. Moreover, we hypothesized that the frequency of Activities Diary usage was associated with worse MFIS-5 and LMSQoL scores at baseline, and better scores at the last assessment.

PwMS who had used the Quickscan at least twice in a period of maximal 6 months, were included in the analyses. For both the MFIS- 5 and the LMSQoL, the difference between the first and last score was tested with paired Student's $t$-tests. Spearman coefficients were calculated for 
the correlations between the frequency of Activities Diary usage, and the MFIS-5 and LMSQoL scores (first score, last score, difference). In line with the explorative character of the analyses in all tests a $P$-value $<0.05$ was considered significant.

\section{Results \\ Development}

The development of the MSmonitor program was practicebased in real life settings with no predefined inclusion or exclusion criteria for participation of PwMS or hospitals. It started in September 2009 in the Medisch Centrum Leeuwarden with the MFIS-5 and LMSQoL questionnaires, both used previously in the online OPTIVIT study ${ }^{6}$ and the Medication and Adherence Inventory. The annual increases in the number of hospitals and PwMS using the program, and in the number of the program's elements are presented in Figure 2. In the period 2009-2014 (April), the numbers of participating hospitals in the consecutive years were 1, 2, $9,9,12$, and 17 ; the number of participating patients were $56,264,422,470$, and 581; and the number of components $4,6,10,10,11$, and 11 .

\section{Participation}

Neurologists and nurses informed PwMS about MSmonitor during outpatient visits. In line with the open, practicebased character of the pilot phase, there were no predefined eligibility criteria for participation. PwMS who agreed to participate in the program were registered online by their neurologist or nurse. Of the 510 PwMS who had been registered as users by March 2013, 470 PwMS had actually started using MSmonitor, with the following characteristics: mean (standard deviation [SD]) age of 47.7 (12.1) years; number of female PwMS 391 (83.2\%) - mean (SD) age 45.8 (12.1) years - and of male PwMS 79 (16.8\%) - mean (SD) age 51.3 (11.1) years (Figure 3).

Nine disciplines of health care professionals used the program, including neurologists $(n=26)$, MS nurses $(n=21)$, physical therapists $(n=9)$, rehabilitation doctors $(n=5)$, continence nurses $(n=2)$, nurse practitioners $(n=2)$, research nurse $(n=1)$, family physician $(n=1)$, and physician assistant $(n=1)$. The multidisciplinary use of the program may prevent unnecessary assessments and improve communication. For example, the neurologist, MS nurse, and continence nurse all have access to the Miction Diary and Miction Inventory outcomes, whereas the continence nurse is responsible for the interpretation of the data and for eventual diagnostic and therapeutic actions; information about patients with abnormal Miction Diary or Miction Inventory findings are safely communicated via the e-consult functionality. The Dutch guidelines for rehabilitation doctors with respect to MS include the MSIP, which is also used by neurologists and MS nurses for their regular outpatient visits; by using the MSmonitor program, the patient is prevented from having to complete the MSIP twice.

\section{Self-management of fatigue}

One-hundred-and-five PwMS met the criteria for being included in the analysis: they had completed the MFIS-5 and LMSQoL questionnaires two or more times in a period of maximal 6 months. Their minimum age was 22.6 years and the maximum age 68.2 years (mean 44.2 [10.6] years). The mean age of males (49.7 [11.2] years) was higher than that of females (mean 43.4 [10.3] years) $(P=0.033)$.

The numbers of PwMS according to sex and frequency of Activities Diary completion are presented in Table 1.

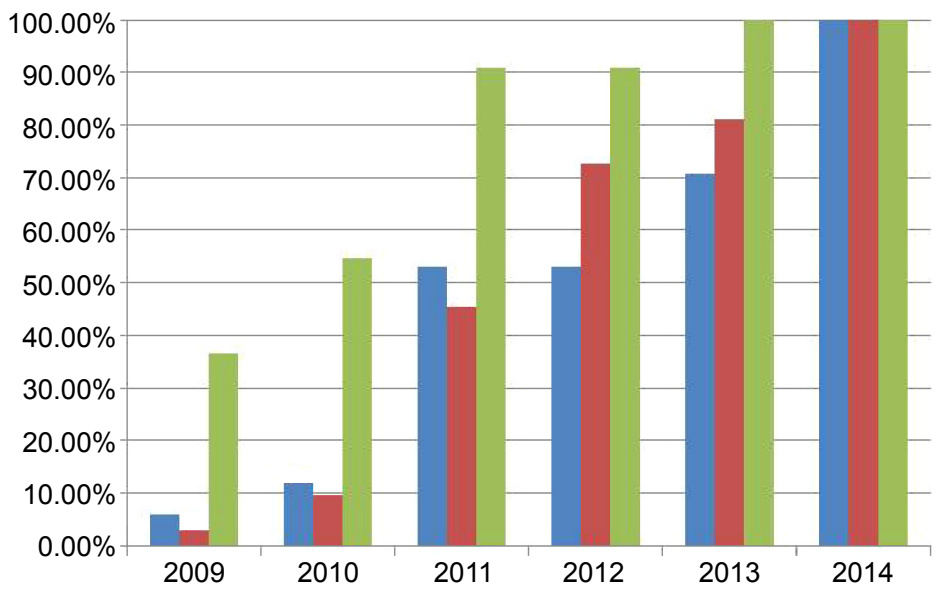

Figure 2 Growth patterns of the number of hospitals (blue bar) and patients (red bar) participating in the MSmonitor program and of the number of program elements (green bar) in the pilot phase from 2009 (0\%) to 2014 (I00\%). 


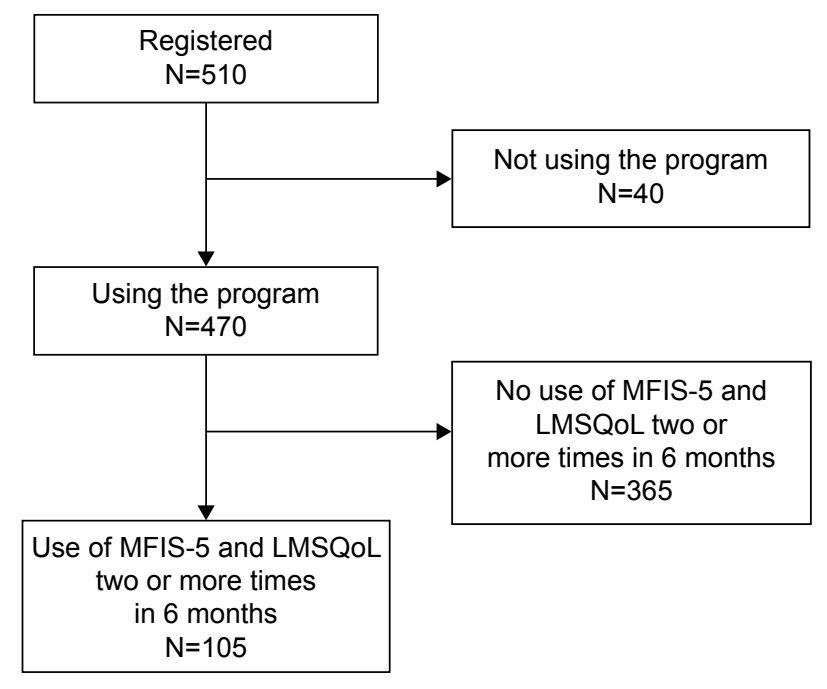

Figure 3 Flow chart showing the numbers of patients registered, using, and not using the MSmonitor program, and the numbers of patients who had used the MFIS-5 and LMSQoL questionnaires two or more times in a 6-month period. Abbreviations: MFIS-5, Modified Fatigue Impact Scale-5; LMSQoL, Leeds Multiple Sclerosis Quality of Life.

The ages in the groups with none, one, and two or more diary completions showed no differences $(P=0.57)$. The interval between the first and the last MFIS-5 and LMSQoL completions ranged between 3 and 183 days (mean 110.8 [52.5] days). The intervals did not differ between the three groups $(P=0.065)$.

The MFIS- 5 scores at first and last assessment, and the difference between the first and last assessment, in the groups with none, one, and two or more diary usages are shown in Table 2.

There were no significant changes over time in MFIS-5 score in either group. The corresponding values of the LMSQoL scores are shown in Table 3.

In all three subgroups the mean LMSQoL score had significantly improved $(P=0.0255, P=0.0147$, and $P=0.0002$, respectively) by $8.97 \%, 12.72 \%$, and $10.06 \%$, respectively. In those PwMS ( $\mathrm{N}=56)$ who had used the Activities Diary at least twice, there was a modest, albeit statistically significant correlation between the frequency of diary usage and the decrease in MFIS-5 score $(r=0.292 ; P=0.028)$. Correlations between diary usage and baseline MFIS- 5 score $(r=-0.123)$ and between diary usage and MFIS-5 score at last assessment ( $r=0.051$ ) were not significant $(P=0.367$ and $P=0.711$, respectively). Similar analyses for the LMSQoL scores yielded no significant correlations.

In all, the findings from these explorative analyses suggest that the self-assessment of fatigue and HRQoL is associated with an increase in HRQoL, and that the use of the Activity Diary may contribute to a decrease in fatigue.

\section{Discussion}

In a period of 5 years we gradually developed a web-based program for self-management and multidisciplinary care in MS, which comprises questionnaires, inventories, diaries, e-consult, and an e-logbook. The questionnaires quantitatively assess MS-related disabilities, perception of disabilities, mental, physical and overall HRQoL, fatigue, anxiety, and depression; the inventories encompass medication, DMD adherence, and urological symptoms; and the diaries record activities and rest periods, and miction and fluid intake.

The demographic characteristics showed that the femaleto-male ratio of the MSmonitor population is almost 5:1 (4.95:1), which is higher than the overall female-to-male ratio in PwMS reported in the literature. In fact, a relatively rapid increase in the sex ratio among PwMS has been reported, ${ }^{29}$ and a meta-analysis of epidemiological studies showed that the worldwide female-to-male ratio of MS did substantially increase over the last century from lower than two to more than three..$^{30}$ In 2013, Boström et al investigated sex ratio trends of MS in Sweden using the National Swedish MS register, and found mean sex ratios of 2.62 in the analysis by year of birth (performed between 1931 and 1985) and 2.57 in the analysis by year of onset (performed between 1946 and 2005). ${ }^{31}$ In New Zealand, studies performed between 1968 and 2001 consistently reported a female-to-male ratio of 2.5:1 to $3: 1 .^{32}$ In all, even the higher end of the increased female-to-male ratio range reported recently, is lower than the

Table I Numbers of PwMS who used the Quickscan (including the MFIS-5 and LMSQoL questionnaires) at least twice in a period of maximal 6 months, according to sex and frequency of Activities Diary completion (0, I or $\geq 2)$

\begin{tabular}{lllll}
\hline Sex & Numbers & & \\
\cline { 2 - 5 } & No completion Activities Diary & One completion Activities Diary & Two or more completions Activities Diary & Total \\
\hline Male & 5 & 1 & 10 & 16 \\
Female & 22 & 21 & 44 & 87 \\
Unknown & 0 & 0 & 2 & 2 \\
Total & 27 & 22 & 56 & 105 \\
\hline
\end{tabular}

Note: For the total group the male-to-female ratio is $1: 5.4$.

Abbreviations: PwMS, persons with multiple sclerosis; MFIS-5, Modified Fatigue Impact Scale-5; LMSQoL, Leeds Multiple Sclerosis Quality of Life. 
Table 2 First and last MFIS-5 scores in a period of maximal 6 months and their difference in Quickscan groups with none, one, and two or more Activities Diary completions

\begin{tabular}{|c|c|c|c|c|c|c|c|}
\hline Activities Diary completions & $\mathbf{N}$ & MFIS-5 score & Mean & SD & Minimum & Median & Maximum \\
\hline \multirow[t]{3}{*}{$\overline{0}$} & 27 & First & 10.48 & 4.34 & 0.00 & 11.00 & 17.00 \\
\hline & & Last & 10.44 & 3.53 & 5.00 & 10.00 & 18.00 \\
\hline & & $\Delta$ & $-0.04^{*}$ & 2.93 & -7.00 & 0.00 & 6.00 \\
\hline \multirow[t]{3}{*}{1} & 21 & First & 12.19 & 5.13 & 1.00 & 12.00 & 20.00 \\
\hline & & Last & 12.24 & 4.74 & 2.00 & 13.00 & 19.00 \\
\hline & & $\Delta$ & $-0.05^{*}$ & 2.87 & -5.00 & -1.00 & 7.00 \\
\hline \multirow[t]{3}{*}{2 or more } & 56 & First & $\mathrm{II} .45$ & 4.11 & 0.00 & 12.00 & 20.00 \\
\hline & & Last & $1 \mathrm{I} .02$ & 4.19 & 0.00 & $1 \mathrm{I} .50$ & 20.00 \\
\hline & & $\Delta$ & $-0.43^{*}$ & 2.53 & -6.00 & 0.00 & 6.00 \\
\hline
\end{tabular}

Notes: Higher scores indicate more fatigue. $* P>0.05$ for comparison between first and last score. $\Delta=$ last minus first score.

Abbreviations: SD, standard deviation; MFIS-5, Modified Fatigue Impact Scale-5.

ratio in the MSmonitor population. So, it seems that female PwMS were either preferentially invited to participate or were more prone to use MSmonitor.

Analysis of the pilot data on fatigue, overall HRQoL, and Activities Diary usage showed results that were in line with the notion that self-management via the MSmonitor program may be beneficial to PwMS: we observed that the use of the two short questionnaires MFIS-5 and LMSQoL was associated with an increase in HRQoL, and that the frequency of Activities Diary usage correlated with the degree of fatigue improvement. In persons with asthma it has been known, for over 2 decades, that guided self-management as part of systematic, planned care can lead to improvements in patient outcomes such as confidence to manage asthma and improved HRQoL, as well as reductions in hospitalizations, emergency room visits, unscheduled visits to the doctor, and days off work or school. ${ }^{33}$ Self-management for MS is a rather recent development, a situation that may partly relate to the variety and complexity of MS symptoms. Fatigue is one of the most frequently reported symptoms, interfering with functioning and resulting in activity limitations and participation restrictions. ${ }^{34}$ As fatigue may also be a side effect of the IFN-beta group of first-line DMDs, it may be difficult for PwMS, neurologists, and nurses to interpret this symptom. The Quickscan, which includes the MFIS-5, LMSQoL, and the Medication and Adherence Inventory, enables monthly assessments of one's experienced fatigue, HRQoL, DMD medication and adherence. In combination with e-education, these tools may give PwMS a better insight into potential relationships between their levels of fatigue, the overall subjective well-being, medication (change) and adherence. PwMS may then adopt a strategy to self-manage their fatigue. Therefore, with the possibilities created by interactive webbased programs, self-management may become a corner stone of disease management in MS.

The data generated online by PwMS are thought to also improve the quality and efficiency of multidisciplinary care. For example, the MSIP provides a complete overview of MS-related symptoms and their subjective valuation, and in MSmonitor the MSIP is completed a few days before an outpatient visit. This has several advantages: PwMS are forced to reflect on their symptoms and thus prepare themselves for

Table 3 First and last LMSQoL scores in a period of maximal 6 months and their difference in Quickscan groups with none, one, and two or more Activities Diary completions

\begin{tabular}{|c|c|c|c|c|c|c|c|}
\hline Activities Diary completions & $\mathbf{N}$ & LMSQoL score & Mean & SD & Minimum & Median & Maximum \\
\hline \multirow[t]{3}{*}{0} & 27 & First & 20.34 & 3.75 & 8.96 & 20.96 & 26.00 \\
\hline & & Last & 21.79 & 3.56 & 8.96 & 21.92 & 26.96 \\
\hline & & $\Delta$ & $1.45^{*}$ & 3.17 & -5.04 & 0.96 & 10.00 \\
\hline \multirow[t]{3}{*}{ I } & 21 & First & 19.15 & 4.32 & 13.04 & 19.04 & 26.96 \\
\hline & & Last & 21.05 & 3.40 & 14.96 & 21.92 & 26.96 \\
\hline & & $\Delta$ & $1.90 * *$ & 3.23 & -4.08 & 2.88 & 7.92 \\
\hline \multirow[t]{3}{*}{2 or more } & 56 & First & 20.38 & 3.62 & 12.08 & 20.00 & 26.96 \\
\hline & & Last & 22.16 & 4.01 & 13.04 & 21.92 & 27.92 \\
\hline & & $\Delta$ & I.78*** & 3.28 & -5.04 & 1.10 & 12.00 \\
\hline
\end{tabular}

Notes: Higher scores indicate more well-being. ${ }^{*} P=0.0255 ;{ }^{* *} P=0.014 \mathrm{I} ;{ }^{*} * *=0.0002 . P$-values for comparisons between first and last score. $\Delta=$ last minus first score. Abbreviations: SD, standard deviation; LMSQoL, Leeds Multiple Sclerosis Quality of Life. 
the onsite consultation; PwMS and professionals can view the completed MSIP questions, the automatically generated domain scores, and their graphic representation prior to the consultation; items and domain scores that have worsened compared to the previous assessment are visualized by a color, and are summarized on the dashboard. The overview of worsened symptoms and their subjective evaluation (disability perception) guides the consultation, and thus the efficiency and quality of multidisciplinary care may improve.

An interesting finding was that of the 385 PwMS who used the Quickscan, 150 (39.1\%) were treated with a DMD. This observation contradicts the opinion that the use of selfassessment programs is biased towards those PwMS who are treated with a DMD, and that persons with no such treatment are less prone to self-assessments due to them having a low disease activity or short disease duration. Moreover, the supposition that persons with progressive MS would not be interested in self-assessments, given their rather predictable disease course, seems not to be in agreement with our data.

Recent developments of MSmonitor include the use by regional networks of qualified professionals for multidisciplinary patient-centered care (MS Care Netherlands), smart phone applications, a self-screening tool for bladder function, the input of neurological data, and data analysis for research purposes. In fact, the combined registration of PROs, neurological and radiological data, and professional health care data seems to qualify the MSmonitor program for being used as (part of) a registry. The efficiency of the MSmonitor program, by using the same PROs for selfmanagement, professional care, and research purposes, is thought to constitute positive feedback for those who are expected to fill the registry with data, and to stimulate PwMS and health care professionals to continue their data input.

Finally, now the pilot phase has ended we will in the next years focus on three main topics: first, a further embedding of the program in the regular care process; second, the development of a 2.0 version and a user's guide, containing eg, information on minimally important differences that represent clinically relevant changes for the various scores; and, third, to analyze the usage by PwMS of the various components and functionalities in relation to demographic, disease, and personal characteristics.

\section{Conclusion}

MSmonitor is an interactive web-based program, developed for self-management and multidisciplinary professional care in MS. Pilot data seem to support the notion that MSmonitor may improve self-management by PwMS.

\section{Acknowledgments}

Members of the MSmonitor Working Group: Ludovicus G Sinnige, Ytsje Dijkstra, Petra Scherstra (Multipele Sclerosis Centre Leeuwarden, Medical Centre Leeuwarden, Leeuwarden, the Netherlands); Björn van Geel, Chantal van Vliet (Department of Neurology, Medical Centre Alkmaar, Alkmaar, the Netherlands); Freek Verheul, Tiny Janssen, Annette Bruinings (Department of Neurology, Groene Hart Hospital, Gouda, the Netherlands); Jiske Fermont, Ron van Dijl, Monique Booy (Amphia Ziekenhuis, Breda, the Netherlands); Wim Verhagen, Gert van Dijk, Helma BorgersDriessen (Department of Neurology, Canisius Wilhelmina Hospital, Nijmegen, the Netherlands); Ruud van der Kruijk, Jorine van Vliet (Department of Neurology, Slingeland Hospital, Doetinchem, the Netherlands); Joost Kerklaan, Sonja de Jong-Stoekenbroek (Department of Neurology, Zuwe Hofpoort Hospital, Woerden, the Netherlands); Hans Schrijver, Marja Lödel, Barbara van Someren-Achterberg (Multiple Sclerosis Centre, Westfries Gasthuis, Hoorn, the Netherlands); Leo H Visser, Vanessa Hens (Multiple Sclerosis Centre Midden Brabant, St Elisabeth Hospital, Tilburg, the Netherlands); Edo P Arnoldus, Lisette Trommelen (Multiple Sclerosis Centre Midden Brabant, Tweesteden Hospital, Tilburg, the Netherlands); Jolijn Kragt, Anita Neele (Department of Neurology, Reinier de Graaf Gasthuis, Delft, the Netherlands); Paul Pop, Thea Bergmans-Barents (Department of Neurology, Viecuri Medical Centre, Venlo-Venray, the Netherlands); Elske Hoitsma, Ilona de Beer, Jolanda van Gorkum (Department of Neurology, Diaconessenhuis Leiden, the Netherlands); Astrid Ruys-van Oeyen, Corinne van den Berg (Department of Neurology, Rijnlandziekenhuis, Leiderdorp, the Netherlands); Erna Bakker, Harry van Leusen, Jur Niewold (Scheper Ziekenhuis, Emmen, the Netherlands). The authors thank the members of the Scientific Advisory Board for their advice.

\section{Disclosure}

Peter Joseph Jongen has received honoraria from Allergan, Biogen-Idec, Merck-Serono, Novartis, sanofi-aventis, and Teva for contributions to symposia as a speaker or for consultancy activities. Anton Kool and Esther van Noort are owners of Curavista bv. The MSmonitor project is funded by TEVA Netherlands via an independent and unrestricted grant. The authors report no other conflicts of interest in this work.

\section{References}

1. Confavreux C, Vukusic S, Moreau T, Adeleine P. Relapses and progression of disability in multiple sclerosis. $N$ Engl J Med. 2000;343(20): $1430-1438$ 
2. Confavreux C, Vukusic S, Adeleine P. Early clinical predictors and progression of irreversible disability in multiple sclerosis: an amnesic process. Brain. 2003;126(Pt 4):770-782.

3. Leray E, Yaouanq J, Le Page E, et al. Evidence for a two-stage disability progression in multiple sclerosis. Brain. 2010;133(Pt 7):1900-1913.

4. Sormani MP, Rio J, Tintore M, et al. Scoring treatment response in patients with relapsing multiple sclerosis. Mult Scler. 2012;19(5):605-612.

5. Rio J, Rovira A, Tintore M, et al. Evaluating the response to glatiramer acetate in relapsing-remitting multiple sclerosis (RRMS) patients. Mult Scler. 2014;20(12):1602-1608.

6. Jongen PJ, Sanders E, Zwanikken C, et al. Adherence to monthly online self-assessments for short-term monitoring: a 1-year study in relapsingremitting multiple sclerosis patients after start of disease modifying treatment. Patient Prefer Adherence. 2013;7:293-300.

7. Harmark L, van Puijenbroek E, van Grootheest K. Intensive monitoring of duloxetine: results of a web-based intensive monitoring study. Eur J Clin Pharmacol. 2013;69(2):209-215.

8. Bandura A. Self-efficacy: toward a unifying theory of behavioral change. Psychol Rev. 1977;84(2):191-215.

9. Bandura A. Social foundations of thought and action: a social cognitive theory. Englewood Cliffs: Prentice-Hall; 1986.

10. Amtmann D, Bamer AM, Cook KF, Askew RL, Noonan VK, Brockway JA. UW-SES: A new self-efficacy scale for people with disabilities. Arch Phys Med Rehabil. 2012;93(10):1757-1765.

11. Stuifbergen AK, Becker H, Blozis S, Timmerman G, Kullberg V. A randomized clinical trial of a wellness intervention for women with multiple sclerosis. Arch Phys Med Rehabil. 2003;84(4):467-476.

12. Motl RW, Snook EM. Physical activity, self-efficacy, and quality of life in multiple sclerosis. Ann Behav Med. 2008;35(1):111-115.

13. e-Study Guide for: Strategies for Teaching Learners with Special Needs. In: Reviews CT, ed. Edition 9; 2012. Available from: https://books. google.nl/books?isbn=1467274461. Accessed August 30, 2015.

14. Wynia K, Middel B, van Dijk JP, de Ruiter H, de Keyser J, Reijneveld SA. The Multiple Sclerosis impact Profile (MSIP). Development and testing psychometric properties of an ICF-based health measure. Disabil Rehabil. 2008;30(4):261-274.

15. Wynia K, Middel B, de Ruiter H, van Dijk JP, de Keyser JH, Reijneveld SA. Stability and relative validity of the Multiple Sclerosis Impact Profile (MSIP). Disabil Rehabil. 2008;30(14):1027-1038.

16. Wynia K, Wijlen AV, Middel B, Reijneveld S, Meilof J. Change in disability profile and quality of life in multiple sclerosis patients: a five-year longitudinal study using the Multiple Sclerosis Impact Profile (MSIP). Mult Scler. 2012;18(5):654-661.

17. Vickrey BG,Hays RD, Harooni R, Myers LW, Ellison GW. A health-related quality of life measure for multiple sclerosis. Qual Life Res. 1995; 4(3):187-206

18. Fisk JD, Ritvo PG, Ross L, Haase DA, Marrie TJ, Schlech WF. Measuring the functional impact of fatigue: initial validation of the fatigue impact scale. Clin Infect Dis. 1994;18 Suppl 1:S79-S83.
19. Ford HL, Gerry E, Tennant A, Whalley D, Haigh R, Johnson MH. Developing a disease-specific quality of life measure for people with multiple sclerosis. Clin Rehabil. 2001;15(3):247-258.

20. Ford HL, Gerry E, Johnson MH, Tennant A. Health status and quality of life of people with multiple sclerosis. Disabil Rehabil. 2001;23(12): 516-521.

21. Zigmond AS, Snaith RP. The hospital anxiety and depression scale. Acta Psychiatr Scand. 1983;67(6):361-370.

22. Tubaro A, Puccini F, De Nunzio C, et al. The treatment of lower urinary tract symptoms in patients with multiple sclerosis: a systematic review. Curr Urol Rep. 2012;13(5):335-342.

23. Bates D, Burks J, Globe D, et al. Development of a short form and scoring algorithm from the validated actionable bladder symptom screening tool. BMC Neurol. 2013;13:78.

24. Burks J, Chancellor M, Bates D, et al. Development and validation of the actionable bladder symptom screening tool for multiple sclerosis patients. Int J MS Care. 2013;15(4):182-192.

25. Kurtzke JF. Rating neurologic impairment in multiple sclerosis: an expanded disability status scale (EDSS). Neurology. 1983;33(11): 1444-1452.

26. Zhang J, Waubant E, Cutter G, Wolinsky JS, Glanzman R. EDSS variability before randomization may limit treatment discovery in primary progressive MS. Mult Scler. 2013;19(6):775-781.

27. Grand'Maison F, Bhan V, Freedman MS, et al. Utility of the Canadian Treatment Optimization Recommendations (TOR) in MS care. Can J Neurol Sci. 2013;40(4):527-535.

28. [Sport MoHWa]. Wet medisch-wetenschappelijk onderzoek met mensen. [Dutch Medical Research Involving Human Subjects Act (WMO)]. [International Publication Series Health, Welfare and Sport]. 1997;(2):1-34. Available from: http://wetten.overheid.nl/ BWBR0009408/geldigheidsdatum_16-10-2015. Accessed August 30, 2015. Dutch.

29. Sadovnick AD. European Charcot Foundation Lecture: the natural history of multiple sclerosis and gender. J Neurol Sci. 2009;286(1-2):1-5.

30. Koch-Henriksen N, Sorensen PS. The changing demographic pattern of multiple sclerosis epidemiology. Lancet Neurol. 2010;9(5):520-532.

31. Boström I, Stawiarz L, Landtblom AM. Sex ratio of multiple sclerosis in the National Swedish MS Register (SMSreg). Mult Scler. 2013;19(1): 46-52.

32. Alla S, Pearson J, Debernard L, Miller D, Mason D. The increasing prevalence of multiple sclerosis in New Zealand. Neuroepidemiology. 2014;42(3):154-160.

33. Lahdensuo A. Guided self management of asthma - how to do it. BMJ. 1999;319(7212):759-760.

34. Oral A, Yaliman A. Revisiting the management of fatigue in multiple sclerosis in the context of rehabilitation: a narrative review of current evidence. Int J Rehabil Res. 2013;36(2):97-104.
Patient Preference and Adherence

\section{Publish your work in this journal}

Patient Preference and Adherence is an international, peer-reviewed, open access journal that focuses on the growing importance of patient preference and adherence throughout the therapeutic continuum. Patient satisfaction, acceptability, quality of life, compliance, persistence and their role in developing new therapeutic modalities and compounds to optimize

\section{Dovepress}

clinical outcomes for existing disease states are major areas of interest for the journal. This journal has been accepted for indexing on PubMed Central. The manuscript management system is completely online and includes a very quick and fair peer-review system, which is all easy to use. Visit http://www. dovepress.com/testimonials.php to read real quotes from published authors. 